\title{
Évaluation de la durabilité socio-économique des ateliers avicoles à M’sila (Algérie)
}

\author{
Nacéra Mahmoudi*, Hacène IkHleF ${ }^{\star *}$, AhCÈne Kaci ${ }^{\star *}$, \\ SOUHILA MAHMOUdI ${ }^{\star \star \star}$
}

DOI: $10.30682 / \mathrm{nm} 1904 \mathrm{e}$

JEL codes: O13, Q01, Q56, R11

\begin{abstract}
Poultry farming contributes to food security, economic development, stability and social equitability for the farmer and his family and supports social bonds. The intervention of these functions together leads to the social and economic sustainability of the farms. In order to determine the degree of sustainability of poultry farms, analyze the strengths and weaknesses of the production system and identify practices to improve we proceeded to assess the socio-economic sustainability of the poultry farms in the province of Msila by the "DIAMOND" method. The results indicate that the best-capitalized farmers and those with technical and economic know-how are aware of the issue of economic viability and diversification of activities and customers, which improves their incomes and decreases the economic fragility of their farms. However, for most farmers, the work is difficult and the productivity of the workforce is low.
\end{abstract}

Keywords: Economic sustainability, Social sustainability, Poultry farming, DIAMOND method.

\section{Introduction}

La viande de volaille est consommée partout dans le monde (Guyomard et al., 2013). Elle bénéficie d'une grande accessibilité en termes économiques et ne fait l'objet d'aucun tabou culturel ou religieux. En outre, elle dispose, auprès des consommateurs, d'une bonne image diététique et sanitaire (Coudurier et Blesbois, 2014). L'accroissement de la consommation des produits avicoles stimule le marché mondial de la volaille qui progresse de 2 à $3 \%$ annuellement (Coudurier et Blesbois, 2013). L'aviculture a été la première production animale à adopter un modèle d'organisation inspiré de l'industrie (lots, bandes d'animaux homogènes, systématisation des opérations,...). Le corollaire en a été un processus général d'intensification de l'élevage (recours aux intrants, recherche de productivité,...) qui a permis des gains spectaculaires de performances zootechniques (Bonaudo et Lossouarn, 2010). Ceci a conduit au développement du modèle de production avicole intensif et à la spécialisation des exploitations avicoles. La production de volaille se fait désormais de manière intensive à $70 \%$ à l'échelle internationale dans les élevages de poulets de chair (Richaud et al., 2015). Néanmoins, les gens s'intéressent plus à la qualité sanitaire, physico-chimique et organoleptique des produits avicoles. Jez (2009)

\footnotetext{
* Université Saad Dahlab de Blida, Blida, Algérie.

** Ecole Nationale Supérieure Agronomique, Département de Production animale, Alger, Algérie.

*** Université de M'sila, Faculté des Sciences, Département des Sciences agronomiques, M'sila, Algérie.

Corresponding author: mahmoudinara@gmail.com
} 
rapporte que les consommateurs augmentent fortement leur demande en oufs dont $70 \%$ de la demande concernerait les productions alternatives (excluant l'élevage en cage) pour des raisons liées au bien-être animal, à l'environnement ou à la santé.

La majorité est d'accord que la suffisance alimentaire, la préservation de l'environnement, la viabilité socio-économique et l'équité sociale sont des composantes importantes de la durabilité (Marta-Costa, 2010). Dans ce contexte, l'aviculture participe à la sécurité alimentaire (approvisionnement régulier des marchés en produits avicoles), au développement économique (création de l'emploi, des revenus et de la richesse), à la stabilité et l'équitabilité sociale pour l'éleveur et sa famille (bon revenu) et soutient les liens sociaux entre acteurs du monde rural (filière courte, partage, foires, associations,...). L'intervention de ces fonctions ensemble conduit à la durabilité sociale et économique de l'exploitation. Celle-ci est renforcée par la pratique de la diversification de la production et des clients.

La réorientation des éleveurs algériens vers la production avicole intensive est devenue une préoccupation majeure de l'Etat, depuis les années 80 , afin d'accroître les disponibilités en protéines animales induites par la croissance démographique, l'urbanisation et la salarisation massive. Dans la wilaya de M'sila (région steppique), le choix de la filière avicole a pour raison le défrichement des parcours, le marché solvable des produits avicoles, la rémunération de l'activité avicole et le coût de production plus faible par rapport aux élevages traditionnels (ovin) qui offrent $82 \%$ du total des viandes rouges produites en 2016. A cela s'ajoute la réticence des jeunes hommes à faire paître les petits ruminants pour des raisons liées aux risques climatiques, conjoncturels et personnels (isolement, incertitudes face à l'avenir, ...). Toutefois, l'activité avicole, dans beaucoup d'élevages, se caractérise par l'irrégularité fonctionnelle notamment dans les exploitations de faible et moyenne tailles en raison des contraintes de différents ordres, ce qui pourrait déstabiliser ces exploitations et les familles qu'elles soutiennent. Afin de déterminer l'impact de ces circonstances sur le fonctionnement de ces exploitations à long terme dans la wilaya d'étude, nous sommes particulièrement intéressés à la question de la durabilité. L'évaluation de la durabilité des ateliers avicoles permet d'identifier les pratiques à améliorer afin d'aider les éleveurs à mieux appréhender le concept de durabilité pour qu'ils puissent l'appliquer facilement à leur échelle.

Pour cela, nous avons adopté la démarche suivante.

\section{Matériel et méthodes}

\subsection{Espace d'investigation et présentation de la zone d'étude}

Les données de l'enquête sont collectées entre 2014 et 2015. L'échantillon choisi aléatoirement s'établit à 15 ateliers avicoles (54 bâtiments d'élevage) toutes filières confondues (poulets de chair, poules pondeuses, dinde et reproducteurs). Ceci représente $5,6 \%$ du total estimé à 968 bâtiments avicoles. Les ateliers retenus se répartissent sur 5 communes de la wilaya de M'sila (Tableau 1). Les éleveurs pris en considération sont ceux qui ont accepté au départ de collaborer et ayant des effectifs dépassant les seuils d'appartenance de 1000 sujets.

Tableau 1 - Localisation des ateliers avicoles enquêtées.

\begin{tabular}{|l|l|c|c|}
\hline Subdivision & Communes & $N^{\circ}$ d'ateliers / région & Fréquence (\%) \\
\hline \multirow{2}{*}{ Magra } & Berhoum & 01 & 6,66 \\
\hline Ouled Derradj & M'sila & 03 & 20 \\
\cline { 2 - 4 } & Maarif & 01 & 6,66 \\
\hline Hammam Dalaa (H. Dalaa) & Maadid & 07 & 46,7 \\
\hline Total & & 03 & 20 \\
\hline
\end{tabular}




\subsection{Choix de la méthode d'évaluation de la durabilité}

Pour l'évaluation de la durabilité des ateliers avicoles de la région d'étude, le choix s'est axé sur la méthode du Diagnostic de durabilité des ateliers d'élevage des Animaux MONogastriques Déclinable par filière " DIAMOND » (Fortun-Lamothe et al., 2011 ; Fortun-Lamothe, 2012). Cette méthode est basée sur une démarche participative de co-construction utilisant, pour la réalisation de ses objectifs, les acteurs de la filière et les acteurs de la recherche et recherche et développement. Cette méthode évalue la durabilité à partir de six objectifs, intégrant les trois échelles de la durabilité (environnementale, sociale et économique) et compte 30 critères au total. Chaque critère comprend un ou plusieurs indicateurs. L'indicateur peut prendre une forme tant quantitative que qualitative et dépend de l'objectif de l'évaluation. Il peut être considéré comme validé s'il est conçu scientifiquement, s'il est sensible et pertinent et s'il est utile et utilisé par les destinataires finaux (Bockstaller et al., 2013 ; Fortun-Lamothe, 2012). La valeur maximale du critère est de 10 points alors que le score attribué à l'indicateur varie de 0 à 10 points selon le poids de ce dernier. La méthode DIAMOND est performante, analyse les forces et les faiblesses du système de production au niveau de l'atelier et permet de proposer des actions pour progresser. Elle dispose de nombreux critères qui sont en équilibre et bien conçus pour répondre aux objectifs essentiels de la durabilité et elle est adaptée à une enquête en temps limité et des aléas conjoncturels (Vilain et al., 2008). Son évaluation dépasse, cependant, l'analyse de la seule performance économique à court terme. En effet, la pérennité d'un système de production dépend d'abord de sa viabilité économique, mais aussi de son indépendance économique, de sa transmissibilité et de son efficience (Zahm et al., 2005). L'échelle de durabilité sociale caractérise, quant à elle, l'insertion de l'exploitation dans son territoire et dans la société et cherche à évaluer la qualité de vie de l'agriculteur et de sa famille ainsi que le poids des services marchands ou non marchands qu'il rend au territoire et à la société (Vilain et al.,
2008). Les critères de l'échelle durabilité environnementale expriment la capacité de l'exploitation à rationaliser ses consommations en ressources non renouvelables avec production de moins de déchets polluants et à valoriser et produire des ressources renouvelables. Cependant, deux critères de cette échelle, production des ressources renouvelables (énergies renouvelables) et utilisation pour production de biomasse (production de la bioénergie) qui permet de réduire les coûts d'énergie et la dépendance aux combustibles fossiles, ne s'adaptent pas au contexte de l'élevage local (pas appliqués) et de ce fait, nous n'avons pas pu évaluer cette échelle (Tableau 2).

\subsection{Les enquêtes}

Le recueil d'informations sur la durabilité des ateliers est mené sous forme d'entretiens semi-structurés auprès des aviculteurs. La dispersion des élevages avicoles et le peu de collaboration de beaucoup d'éleveurs ont limité l'élargissement de l'échantillon. L'évaluation de la durabilité des élevages avicoles implique la détermination de l'ensemble des conditions de fonctionnement des ateliers afin de pouvoir identifier leurs caractéristiques structurelles, la diversité de leurs pratiques agricoles et leurs performances zootechniques. A cet effet, un questionnaire détaillé de 104 questions a été élaboré pour faciliter la collecte des données. Celui-ci est subdivisé en deux parties : la première partie comprend 42 questions qui donnent des informations sur l'identification des exploitations avicoles et la deuxième partie du questionnaire comprend 62 questions (indicateurs) pour le recueil des informations nécessaires au calcul des critères de la durabilité. Ces critères sont regroupés en deux échelles : échelle économique (15 indicateurs) et échelle sociale (47 indicateurs) (Annexe 1).

\subsection{Analyse statistique des données}

Les données collectées ont subi une analyse descriptive à l'aide du logiciel Excel pour déterminer les caractéristiques générales des exploitations avicoles. Une analyse de la variance à une 
Tableau 2 - Objectifs et critères de la durabilité socio-économique.

\begin{tabular}{|c|c|c|c|}
\hline Echelle & Objectif général & Critères & Note (points) \\
\hline \multirow{10}{*}{$\begin{array}{l}\text { Economie : } \\
100 \text { points }\end{array}$} & \multirow{5}{*}{$\begin{array}{l}\text { Économiquement } \\
\text { rentable } \\
\text { (50 points) }\end{array}$} & Viabilité économique & 10 \\
\hline & & Efficacité main d'œuvre & 10 \\
\hline & & Efficience du processus productif & 10 \\
\hline & & Indépendance technique & 10 \\
\hline & & Rentabilité économique & 10 \\
\hline & \multirow{5}{*}{$\begin{array}{l}\text { Flexible et } \\
\text { adaptable } \\
\text { (50 points) }\end{array}$} & Spécialisation économique & 10 \\
\hline & & Sensibilité aux aides & 10 \\
\hline & & Autonomie financière & 10 \\
\hline & & Transmissibilité & 10 \\
\hline & & Polyvalence de l'outil de production & 10 \\
\hline \multirow{10}{*}{$\begin{array}{l}\text { Sociale : } \\
100 \text { points }\end{array}$} & \multirow{5}{*}{$\begin{array}{l}\text { Préserve la } \\
\text { qualité de vie et } \\
\text { les conditions } \\
\text { de travail du } \\
\text { producteur ( } 50 \\
\text { points) }\end{array}$} & Viabilité socio-économique & 10 \\
\hline & & Repos et organisation du temps & 10 \\
\hline & & Pénibilité du travail & 10 \\
\hline & & Implication dans le milieu & 10 \\
\hline & & Insertion dans la vie locale & 10 \\
\hline & \multirow{5}{*}{$\begin{array}{l}\text { Répond aux } \\
\text { demandes } \\
\text { du citoyen- } \\
\text { consommateur } \\
\text { ( } 50 \text { points) }\end{array}$} & Qualité et traçabilité & 10 \\
\hline & & Pratiques d'élevage respectueuses du bien-être animal & 10 \\
\hline & & Milieu de vie respectueux du bien-être animal & 10 \\
\hline & & Emploi Filière Courte & 10 \\
\hline & & Services non-agricoles & 10 \\
\hline
\end{tabular}

Source : Fortun-Lamothe (2012).

voie «ANOVA » a été effectuée pour l'appréciation des corrélations statistiques (r) par l'utilisation du logiciel « MINITAB 15 » et une analyse en Composantes Principales (ACP) pour élaborer la typologie de la durabilité des exploitations par l'utilisation du logiciel « Past 1.8 ».

\section{Résultats et discussion}

\subsection{Identification des exploitations avicoles enquêtées}

Les élevages avicoles sont conduits par des jeunes éleveurs ( $44 \pm 18$ ans) dotés d'une expérience de 3 à 17 ans. Les bâtiments sont de type industriel dont $70,4 \%$ en dur et $29,6 \%$ des tun- nels (serres en plastiques munies d'isolation et destinés à la production de poulets de chair). La taille des exploitations varie d'un maximum de 60 ha à un minimum d' 1 ha $(\mathrm{CV}=150 \%)$ avec une superficie moyenne de 10 ha. L'atelier d'élevage comprend en moyenne $4 \pm 2$ bâtiments de $697 \mathrm{~m}^{2}$ en moyenne.

Le 33,3\% des exploitations se caractérisent par une diversité animale (volailles, ovin, bovin et abeille) tandis que la diversité avicole (poulet, dinde, poules,...) est observée notamment dans les exploitations de grandes tailles $(23,7$ ha et 5,3 bâtiments de $785 \mathrm{~m}^{2}$ ). Les élevages enquêtés sont de type standard (poulet, dinde et reproducteurs sont au sol alors que les poules pondeuses sont en cages conventionnelles). La taille des ef- 
fectifs de volailles exploités est très divergente $(\mathrm{CV}=63,2 \%$ ), elle varie de 2000 sujets (élevage de la dinde) à 36600 sujets (élevage des poulettes). Les souches les plus exploitées sont l'Arbor acres $(30,4 \%)$, l'ISA15 $(30,4 \%)$ et la Coob 500 (21,7\% du total). Pour l'élevage de la dinde, la souche «Big Aviagen » est la plus utilisée.

En revanche, la diversité végétale est représentée essentiellement par la céréaliculture (blé dur et orge) et l'arboriculture (abricotiers,...) qui sont pratiquées respectivement par 26,7 et $53,3 \%$ des exploitants ; le reste $(20 \%)$ ne pratique aucune culture. La diversité de l'activité (production animale et végétale) au sein de l'exploitation est un paramètre de la multifonc- tionnalité qui constitue, selon Guillaumin et al. (2009), un moyen d'aller vers un développement durable.

\subsection{Evaluation de la durabilité}

\subsubsection{L'échelle de durabilité économique}

Le score total de la durabilité économique des ateliers enquêtés s'établit à 65,4 points $(\mathrm{CV}=$ $17,12 \%$ ) sur un score théorique de 100 points au maximum (Figure 1). La quasi-totalité des exploitations présente de bonnes pratiques pour ce qui concerne la rentabilité économique, la sensibilité aux aides et l'autonomie financière.

Figure 1 - Durabilité économique moyenne des ateliers avicoles.

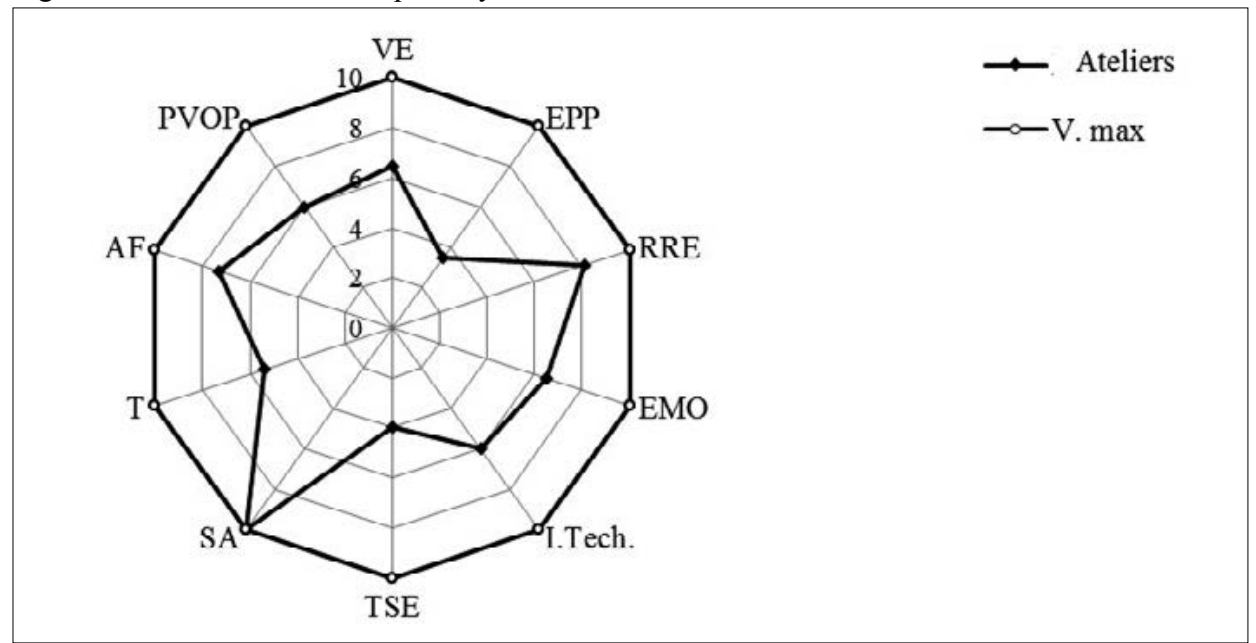

$V E$ : viabilité économique; EPP : efficience du processus productif; RRE : ratio de rentabilité économique ; EMO : efficacité de la main-d'œuvre ; I.Tech : Indépendance technique ; TSE : taux de spécialisation économique ; $S A$ : sensibilité aux aides ; $T$ : transmissibilité ; AF : autonomie financière ; PVOP : polyvalence de l'outil de production ; V. max : valeur maximale.

Les indicateurs efficience du processus productif $(3,47$ points $)$ et taux de spécialisation économique (4 points) sont mal notés dans la plupart des ateliers. La faible efficience exprime l'importance de la dépendance de ces ateliers aux intrants, ce qui diminue leur autonomie et leur économie des ressources alors que le taux de spécialisation économique élevé $(90,9 \pm 15,4 \%)$ indique un manque d'adaptabilité et de cohérence.
L'insuffisance dans la diversification des sources de revenus (activités et clients) rend ces ateliers plus fragiles face aux contraintes économiques (évolution des marchés, prix des intrants,...), cumulant ainsi les facteurs de risque. Celle-ci peut s'expliquer par le type des transactions qui existent entre les éleveurs et les fournisseurs d'intrants d'une part, et, d'autre part, les éleveurs qui vendent leurs produits souvent en gros à celui qui 
offre le meilleur prix sans s'intéresser à la question de la diversification des clients.

La durabilité économique la plus élevée ( $\geq 69 \%$ du score total) est à l'actif de 6 ateliers (E1 et E11 avec 78 points, E3 et E6 avec 74 points et E5 et E8 avec 73 points) (Figure 2). Ces ateliers se caractérisent par une bonne performance économique ( $R R>35 \%$ ) et sont viables économiquement car leurs capitaux investis dégagent une bonne capacité d'autofinancement. Ils se caractérisent également par une bonne effi- cacité de la main-d'œuvre, une faible sensibilité aux aides et une meilleure polyvalence de l'outil de production.

Les ateliers fragiles économiquement (E10 et E12) ne profitent pas suffisamment de leurs capitaux investis qui ne génèrent consécutivement que 10,4 et 25,8 DA par 100 DA investis et se caractérisent aussi par une faible diversification de leur activité. Ces deux éleveurs sont les plus décapitalisés (faible taille d'élevage, manque de moyens de financement,...).

Figure 2 - Durabilité économique des ateliers avicoles enquêtés.

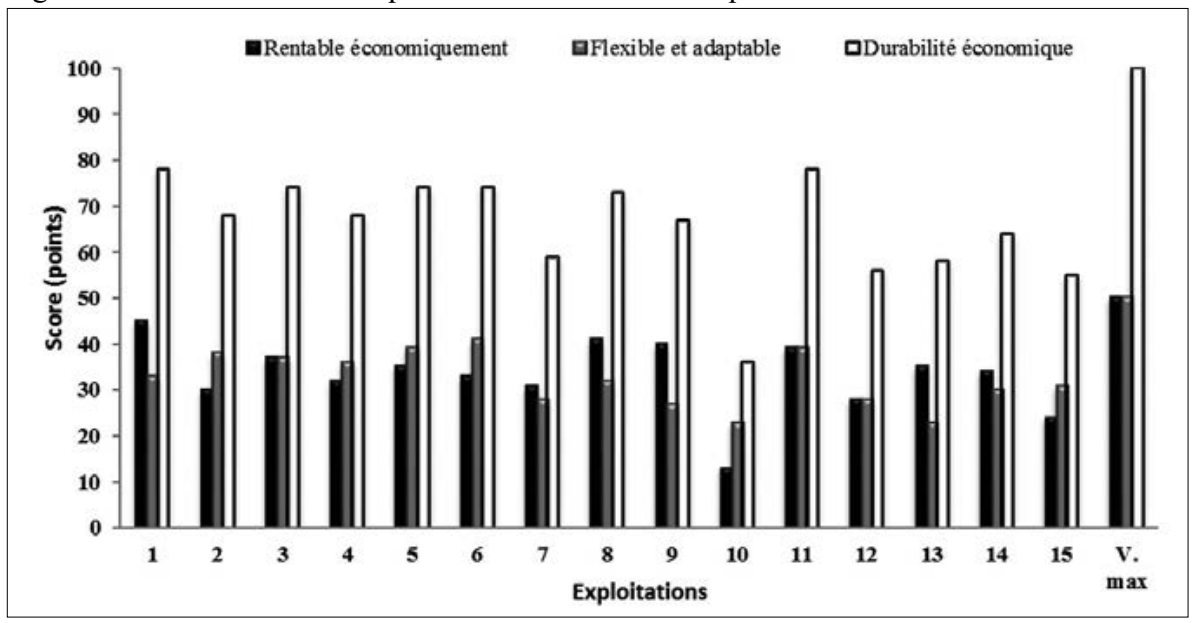

Figure 3 - Durabilité sociale de toutes les ateliers avicoles enquêtés.

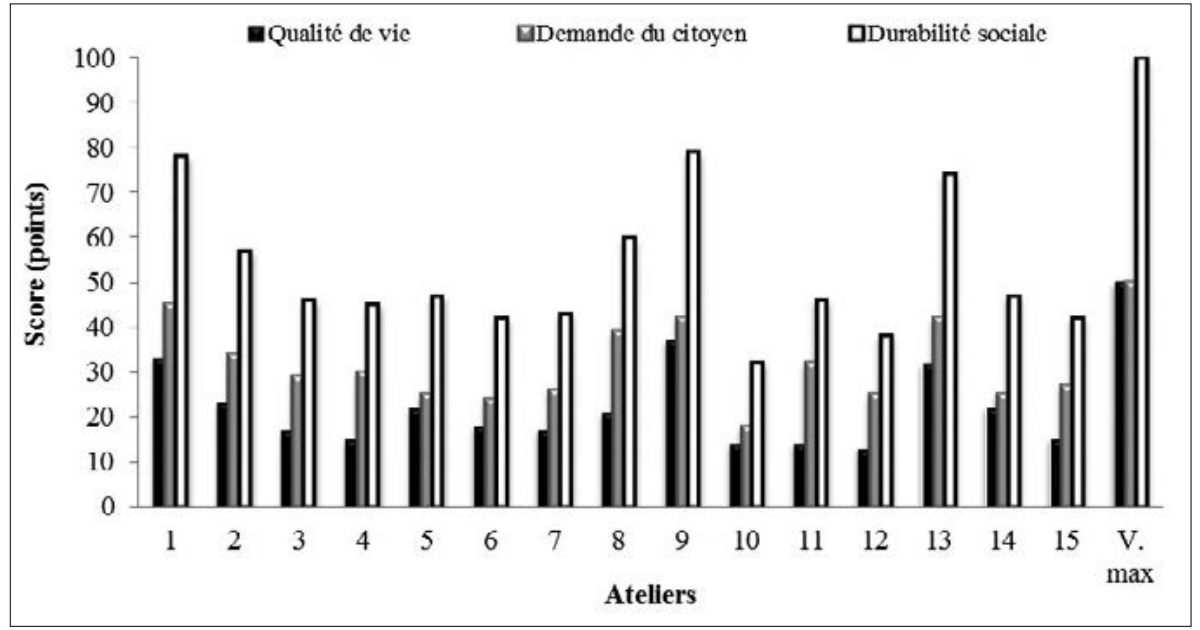




\subsubsection{L'échelle de durabilité sociale}

La durabilité sociale moyenne des ateliers s'établit à 51,73/100 points. Les ateliers E9, E1 et $\mathrm{E} 13$ se révèlent les plus socialement équitables avec respectivement 79, 78 et 74 points (Figure 3 ). Ceux-ci répondent bien aux demandes du citoyen-consommateur. Ces ateliers se caractérisent par un niveau de capitalisation important, ce qui permet aux exploitants d'exercer leurs activités dans des conditions favorables et de mener une vie descente avec leurs familles.

Dans l'ensemble, la durabilité sociale est pénalisée par la qualité de vie et les conditions de travail du producteur. Les scores des critères de cet objectif varient de 13 à 37 points avec une moyenne de 20,9 \pm 7,6 points pour un maximum théorique de 50 points. Les ateliers les plus viables socio-économiquement (E1, E8 et E9) (20\% du total) enregistrent des scores allant de 6 à 7 points. Ceux-ci se caractérisent par un tonnage important des produits mis sur le marché, des prix proposés compétitifs, un nombre important de points de vente et une bonne adaptabilité aux fluctuations du marché (recherche de la clientèle, réduction des prix, vente par facilité...).

La moyenne des scores du critère «Insertion dans la vie locale » est très faible (2 points). Toutefois, 4 ateliers (E1, E8, E9 et E13) ont des notations acceptables, allant de 6 à 7 points car ils participent aux foires locales et même aux documentaires télévisés.

Figure 4 - Durabilité sociale moyenne des ateliers avicoles.

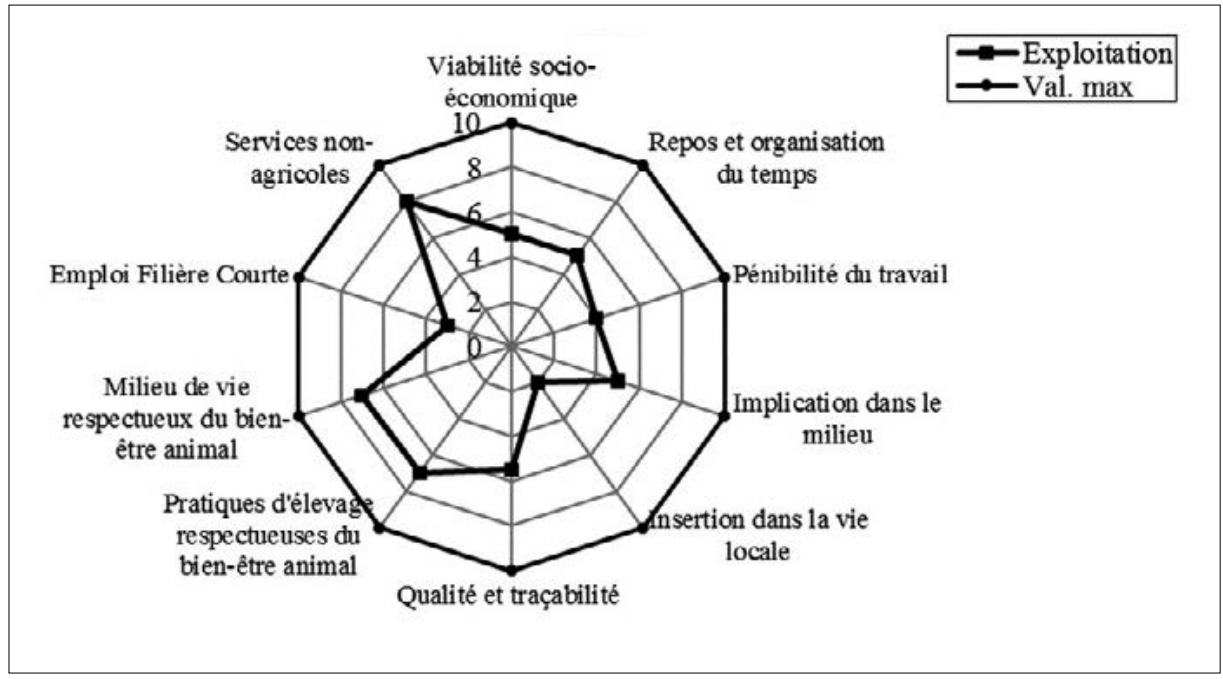

Le critère pénibilité du travail est sanctionné par une note moyenne de $4 / 10$ points car $66,7 \%$ des ateliers enregistrent des scores inférieurs à 5 points. Ceci est dû particulièrement au travail pénible mené par le chef d'exploitation qui supervise toutes les tâches du travail, ainsi qu'à la faible productivité de l'unité de travail humain (UTH) par $\mathrm{m}^{2}$ qui dépasse largement la norme mentionnée dans les élevages industriels. Dans cette optique, Gallot (2011) indique que d'après les estimations empiriques couramment utilisées, une unité de main-d'œuvre peut prendre en charge 3500 à $4000 \mathrm{~m}^{2}$ de poulets standards ou de dindes. La faible productivité est un facteur de mauvaise gestion qui diminue la rentabilité. Le manque de mécanisation dans certains bâtiments et la faible taille du parquet d'élevage peuvent expliquer la faible productivité. Les notations du critère repos et organisation du temps varient de 2 à 8 points (4,8 points en moyenne). Les scores minimums 
sont inscris chez les exploitants travaillant péniblement (pas de week-ends et vacances) tandis que les ateliers de forme sociétaire qui disposent de différents services administratifs organisant le travail se caractérisent par des scores élevés.

Les ateliers E9, E1, E13 et E8, bien impliqués socialement, ont des notations respectives de 10 , 8,8 et 7 points. Ces scores sont en relation avec la pratique de la pluriactivité (élevage, accouvage, abattage, vente des intrants,...), l'accueil des étudiants pour des stages de fin d'étude, le niveau de formation élevé des exploitants et leur engagement dans les travaux humanitaires (offrent des dons aux associations civiles caritatives et aux personnes fragiles, construction des écoles,...).

Les pratiques d'élevage respectueuses du bien-être animal (accès suffisant aux mangeoires, aux abreuvoirs et à l'eau propre, mécanisation de la chaîne de production, confort et bon état physique du cheptel), le milieu de vie respectueux du bien-être animal et la disponibilité des services non-agricoles au niveau de leur région d'habitat (logements, services sanitaires, réseau d'électricité et de gaz, eau potable, transport et écoles) sont les critères responsables de la bonne notation de l'objectif « répond aux demandes du citoyen consommateur ». Celle-ci s'établit en moyenne à 29,9 points $(59,6 \%$ du total). Toutefois, les deux critères « qualité et traçabilité » $(5,5$ points) et « emploi filière courte » $(2,9$ points $)$ sont mal représentés dans la plupart des ateliers. Le faible score du critère qualité et traçabilité est lié à l'absence de la proposition des produits naturels et locaux et la qualité différenciée des produits (absence d'une agriculture biologique et des produits labels, taux d'incorporation bas des matières locales dans l'aliment,...). Ceci corrobore avec les travaux de Ghozlane et al. (2006) qui constatent une absence du critère « qualité et traçabilité» dans les exploitations bovines laitières de la Wilaya de Tizi-Ouzou (Algérie). En revanche, les ateliers E1, E6, E8, E9, E11 et E13 disposant d'une stratégie d'intégration de la chaîne de valeur ont enregistré des scores plus élevés (6 à 7 points).

Ces ateliers sont aussi performants dans la qualité d'usage ou de service grâce à leurs pro- duits portant le logo de l'atelier et les étiquettes. Pour l'indicateur Emploi, la note maximale de 4 points a été attribuée aux ateliers qui participent activement à la création de l'emploi. Toutefois, les deux autres indicateurs représentant la filière courte (circuits de commercialisation agro-industrielle et formes de vente) sont mal signalés dans la plupart des ateliers car les producteurs vendent leurs produits en gros ou demi-gros aux commerçants qui revendent à leur tour les produits aux intermédiaires.

Le score du critère « Emploi Filière Courte » s'établit en moyenne à 2,93 points $(29,3 \%$ du score total). La note maximale de 4 points a été attribuée aux ateliers qui participent activement à la création de l'emploi (E1, E3, E8, E9, E11 et E13). Toutefois, la filière courte (circuits de commercialisation agro-industrielle et formes de vente) est faiblement représentée dans la plupart des ateliers puisque les éleveurs vendent leurs produits à travers les intermédiaires. L'atelier E1 enregistre, quant à lui, le score maximum de 6 points grâce à la vente directe au consommateur ou à la restauration collective par le biais d'un seul intermédiaire ainsi qu'à la transformation des produits de la ferme pour la vente (abattage). Ceci permet d'après, Chiffoleau (2010), d'augmenter le lien social, offrir des produits frais et établir des prix transparents et raisonnables, sans marges pris par les intermédiaires. Dans d'autres ateliers, le circuit court concerne notamment les produits secondaires de l'exploitation (moutons, veaux, lait, D'han, abricots, olives,...) qui sont vendus directement aux consommateurs.

Dans l'ensemble, nos résultats sont confirmés par Yakhlef et al. (2008) qui rapportent que la durabilité socio-territoriale des exploitations bovines laitières en zone semi-aride est pénalisée par des scores moyens ou très faibles à cause de l'inexistence de démarches de traçabilité ou de dispositifs pour homologuer les produits, l'absence de programmes de formation adaptés aux niveaux scolaires des éleveurs, une faible implication des éleveurs dans les structures associatives, une faible valorisation des produits par filières courtes et un temps de travail par actif (éleveur) important. 


\subsection{Etude typologique de la durabilité des ateliers étudiés}

\subsubsection{Définition des axes}

La contribution cumulée à l'inertie totale des informations contenues dans les deux premiers axes factoriels retenus est de 57,01\% (Tableau 3).

\subsubsection{Définition des classes d'éleveurs}

L'analyse multivariée, effectuée sur les 15 ateliers enquêtés, a mis en évidence quatre classes bien distinctes du point de vue durabilité des ateliers, pratiques, performances zootechniques, diversité des espèces animales sur l'exploitation et caractéristiques socioprofessionnelles des éleveurs (Tableaux 4 et 5 et Figures 5 et 6 ).

Tableau 3 - Valeurs propres et leur contribution à l'inertie des axes factoriels.

\begin{tabular}{|c|c|c|c|}
\hline Axes factoriels & Valeurs propres & \% d'inertie & \% cumulé \\
\hline 1 & 3,314 & 33,14 & 33,14 \\
\hline 2 & 2,387 & 23,87 & 57,01 \\
\hline 3 & 1,52 & 15,21 & 72,21 \\
\hline
\end{tabular}

Tableau 4 - Variance des ateliers sur les principaux axes.

\begin{tabular}{|c|c|c|c|c|}
\hline Classe & Ateliers & Axe 1 & Axe 2 & Axe 3 \\
\hline 1 & 1 & $-2,679$ & 1,697 & $-2,494$ \\
\hline \multirow{3}{*}{3} & 13 & 0,2479 & 3,165 & 0,548 \\
\cline { 2 - 5 } & 14 & 0,0954 & 0,67 & $-0,079$ \\
\cline { 2 - 5 } & 2 & 0,3341 & 0,083 & $-0,079$ \\
\cline { 2 - 5 } & 3 & $-0,574$ & $-2,08$ & 0,152 \\
\cline { 2 - 5 } & 7 & 1,2531 & $-1,19$ & 0,999 \\
\cline { 2 - 5 } & 8 & $-1,186$ & $-1,32$ & $-1,343$ \\
\cline { 2 - 5 } & 10 & 2,8776 & 1,075 & 1,136 \\
\cline { 2 - 5 } & 11 & 0,5306 & 0,245 & $-1,544$ \\
\cline { 2 - 5 } & 12 & 1,7906 & 0,17 & 0,207 \\
\hline \multirow{6}{*}{4} & 15 & 1,6539 & 0,736 & 0,324 \\
\cline { 2 - 5 } & 4 & 0,9566 & 0,161 & $-0,951$ \\
\cline { 2 - 5 } & 5 & $-0,373$ & $-1,89$ & $-0,029$ \\
\cline { 2 - 5 } & 9 & $-0,461$ & $-2,5$ & 0,523 \\
\hline
\end{tabular}

La classe 1 qui comprend un seul atelier (E1) $(6,7 \%$ du total) est représentée en valeur positive sur l'axe 2. Cet atelier est spécialisé dans l'élevage de poulets de chair. Il est meilleur dans la durabilité socio-économique par rapport aux trois autres classes avec respectivement 78 et 78 points. Il enregistre, en outre, de bonnes perfor- mances zootechniques (indice de consommation et mortalité bas,...).

De taille moyenne (10 ha), cet atelier se caractérise toutefois par l'absence de diversité animale. Il dispose de potentialités de production importantes (effectif animal annuel élevé « 325000 sujets », unité d'aliment du bétail, 
abattoir industriel performant et bien équipé, humains non salariée (père et fils). Cet atelier chambre froide, des locaux de boucheries, des camions frigorifiques pour la distribution de la viande et des camions aménagés pour le transport des poussins) et d'une technologie de pointe qui est très bien maîtrisée par l'unité du travail possède un marché très diversifié (propres locaux de boucheries, cantines de l'université, hôpitaux, casernes militaires,...) et ses produits sont distribués avec des étiquettes au logo de l'entreprise.

Tableau 5 - Caractéristiques moyennes des ateliers selon la classe d'éleveurs.

\begin{tabular}{|l|c|c|c|c|}
\hline Paramètres & Classe 1 & Classe 2 & Classe 3 & Classe 4 \\
\hline Ateliers & 1 & $13 ; 14$ & $2 ; 3 ; 7 ; 8 ; 10 ; 11 ; 12 ; 14 ; 15$ & $4 ; 5 ; 6 ; 9$ \\
\hline Nombre & 1 & 2 & 8 & 4 \\
\hline Fréquence (\%) & 6,7 & 13,3 & 53,3 & 26,3 \\
\hline D.soc & 78 & 60,5 & 45,5 & 53,25 \\
\hline D.Econo & 78 & 57,5 & 62,38 & 70,5 \\
\hline TM\% & 10 & 6,5 & 10,55 & 10,13 \\
\hline IC & 2,08 & 2,35 & $2,6 * ; 236,9 * *$ & 2,26 \\
\hline UTHNS & 3 & 0,5 & 1,5 & 3,25 \\
\hline Ha & 10 & 3,5 & 6,56 & 17,5 \\
\hline NBât & 5 & 4 & 2,25 & 5,25 \\
\hline D.avic & 1 & 1,5 & 1,63 & 2,25 \\
\hline D.ani & 0 & 0 & 0,5 & 0,5 \\
\hline Eff.an & 325000 & 200000 & 18025 & 51375 \\
\hline U.Prod & 6 & 0 & 1,38 & 1,5 \\
\hline
\end{tabular}

D.soc : durabilité sociale ; D.Econo : durabilité économique ; TM\% : taux de mortalité (\%) ; IC : indice de consommation ; UTHNS : unité de travail humain non salarié; Ha : superficie (ha); NBât : nombre de bâtiment ; D.avic : diversité avicole ; D.ani : diversité animale ; Eff.an : effectif animal ; U.Prod : unité de production; * poulet; ** pondeuses.

Figure 5 - Arbre de classification hiérarchique ascendante (liaison de Ward).

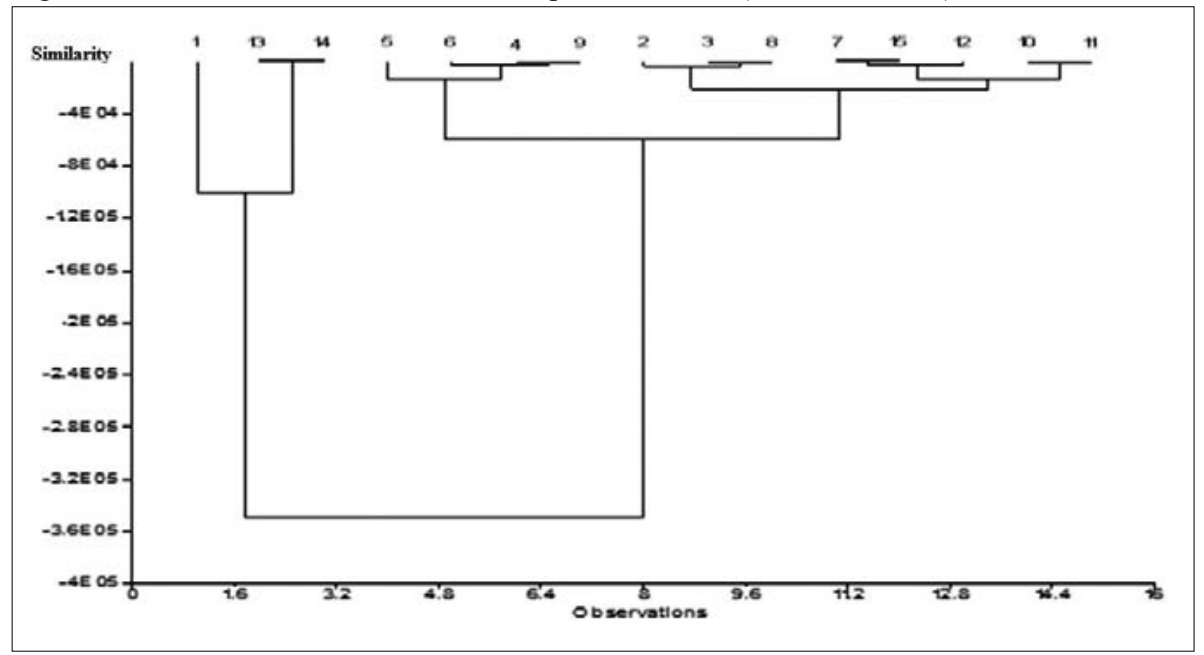


La classe 2 qui regroupe deux ateliers avicoles E13 et E14 enregistre le taux de mortalité le plus bas $(6,5 \%)$. Ces ateliers se caractérisent par une taille d'élevage importante et une absence de diversité d'élevage (poulette démarrée pour E13 et poulet de chair et dinde pour E14). La durabilité économique et sociale de ces ateliers, représentées négativement sur l'axe 2 , sont acceptables (60 et 61 points respectivement).

La classe 3 rassemble 8 éleveurs $(53,3 \%$ du total) projetés en majorité positivement sur l'axe 1. Cette classe regroupe principalement les éleveurs du poulet de chair et de dinde $(73,3 \%$ $\mathrm{du}$ total) et secondairement les éleveurs de poules pondeuses $(26,7 \%)$ qui enregistrent les plus faibles performances zootechniques (taux de mortalité et indice de consommation élevés). Les ateliers de cette classe sont faiblement durable socialement car les éleveurs travaillent durement dans de petits ateliers décapitalisés (18025 sujets/an dans 2,25 bâtiments/exploitant). Ils se distinguent par une mauvaise insertion dans la vie locale. Leur cursus qualité-traçabilité est également faible. Toutefois, ils présentent une diversité dans l'élevage avicole et dans l'élevage des autres animaux.

La quatrième classe (4 éleveurs, soit 26,3\% de l'échantillon) regroupe les ateliers spécialisés dans l'élevage des reproducteurs et de poules pondeuses. Ces derniers sont projetés positivement sur l'axe 1. Ces élevages sont de grandes tailles (17,5 ha, 5,25 bâtiments et 51375 pondeuses/an), bien capitalisés et disposent d'autres unités de production (unité d'aliment du bétail, couvoirs, pharmacie des produits vétérinaires). Ils se caractérisent également par une diversité avicole et une diversité animale (élevage ovin, bovin,...). Ces ateliers sont économiquement durables (70,5 points) mais socialement non équitables à l'exception de l'atelier E9 (79 points).

Figure 6 - Projection des ateliers sur les axes factoriels 1 et 2.

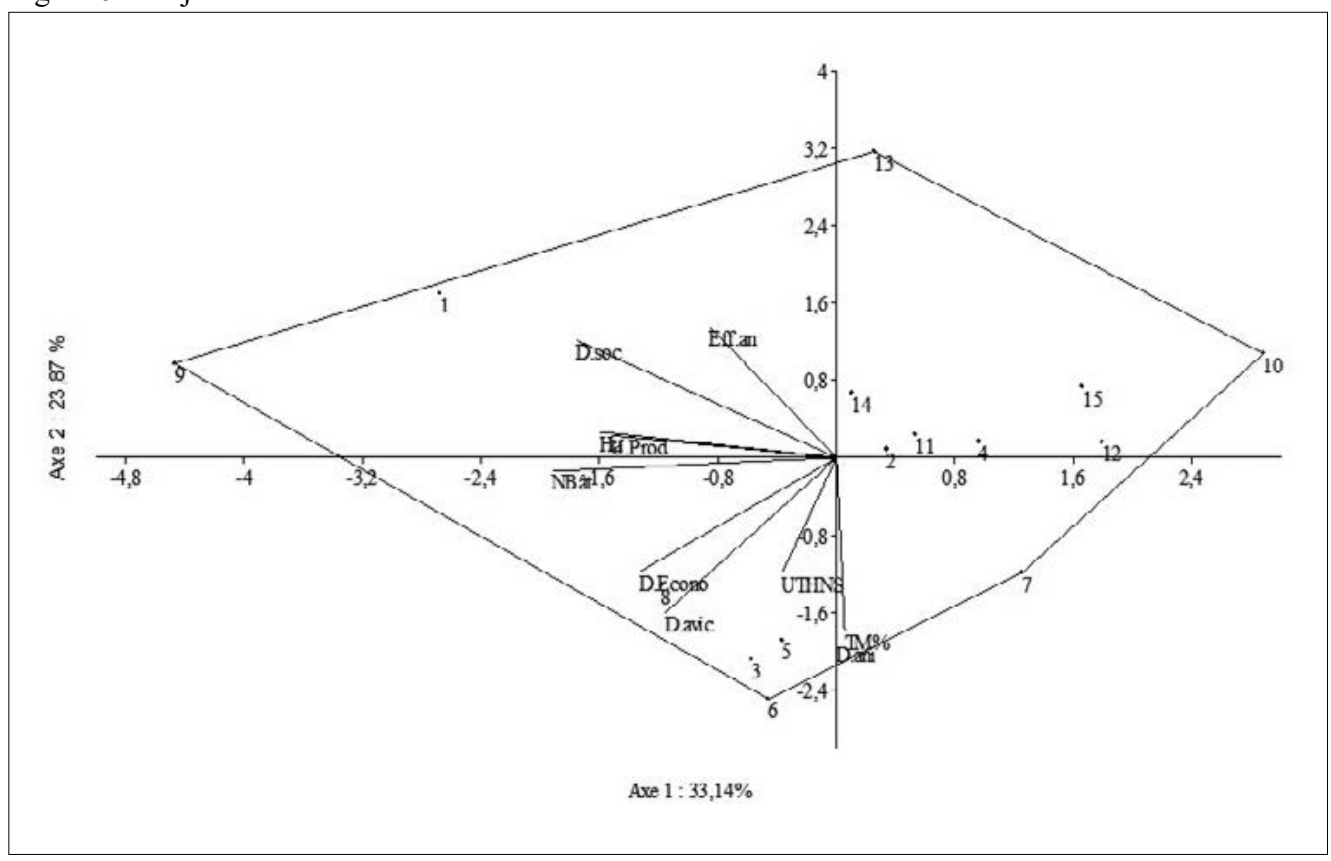

D.soc : durabilité sociale ; D.Econo : durabilité économique ; TM\% : taux de mortalité (\%);

$I C$ : indice de consommation ; UTHNS : unité de travail humain non salarié ; Ha : superficie (ha) ; NBât : nombre de bâtiment ; D.avic : diversité avicole ; D.ani : diversité animale; Eff.an : effectif animal ; U.Prod : unité de production. 


\section{Conclusion}

Le concept de durabilité est aujourd'hui une préoccupation centrale au sein du secteur agricole. La démarche de conception ne peut être dissociée de la démarche d'évaluation. Becker (1997) résume cette situation par la formule « be honest, be modest, be clear and be cautious » pour souligner que l'évaluation sera d'autant plus efficace que les objectifs seront clarifiés et que les normes et les valeurs qui la sous-tendent sont clairement exposées.

L'exploration des résultats de la durabilité socioéconomique des ateliers avicoles de la wilaya d'étude montre que les aviculteurs sont conscients de la question de la durabilité économique $(65,4 / 100$ points $)$ car la quasi-totalité des ateliers ont de bonnes pratiques dans la sensibilité aux aides et l'autonomie financière et leurs capitaux investis dégagent une bonne capacité d'autofinancement. Toutefois, les critères « efficience du processus productif » et « taux de spécialisation économique » sont mal représentés dans la plupart des ateliers (dominance d'une activité par rapport aux autres). La durabilité de ces ateliers est limitée par l'échelle sociale $(51,7 / 100$ points) qui est pénalisée par la qualité de vie des exploitants, la faible productivité et la mauvaise insertion dans la vie locale. Ainsi, l'Emploi Filière Courte est restreinte par la présence d'intermédiaires, ce qui diminue le lien social.

Pour les ateliers fragiles économiquement (E10 et E12), ils devraient bien profiter de leurs capitaux investis qui ne génèrent que 10,4 et 25,8 DA par 100 DA investis et augmenter la taille de leurs ateliers par des crédits bancaires. Ainsi, ils devraient intervenir sur le rétablissement des indicateurs des critères d'autonomie financière et du taux de spécialisation économique par l'introduction d'autres espèces animales par la diversification des modes d'élevage.

Les éleveurs des classes 1 et 2 enregistrant des performances acceptables sont meilleures dans la durabilité socio-économique (78 points) mais doivent diversifier leurs activités d'élevage (avicole et autres). Toutefois, les éleveurs de la classe 3 qui sont faiblement durable socialement doivent s'investir dans l'accroissement de la taille de leurs exploitations, la gestion de la santé des animaux et l'aménagement de leurs bâtiments et la bonne organisation du travail pour améliorer les performances zootechniques et rentabiliser leurs activités.

En termes de perspectives pour sa durabilité dans les régions steppiques, toutes les exploitations avicoles devraient se regrouper dans des associations et des collectivités pour défendre leurs intérêts communs et augmenter le lien social et l'échange de savoir, des techniques et du matériel de production. Ainsi, l'introduction des produits de qualité et l'optimisation des produits locaux contribuent à la préservation de l'identité du territoire des producteurs et améliorent la diversification des produits et des revenus pour attirer les consommateurs et les investisseurs et notamment les jeunes.

Il paraît nécessaire d'utiliser la méthode DIAMOND, avec tous ses critères, dans l'évaluation de la durabilité des exploitations des différentes filières avicoles et de comparer les résultats obtenus afin d'établir des indicateurs pertinents et améliorer cette méthode pour faciliter son utilisation par les chercheurs, les étudiants et les aviculteurs.

La question de l'environnement des élevages avicoles est ainsi très intéressante. Elle ne concerne pas uniquement l'aviculteur mais aussi tous les acteurs représentés par les inspections vétérinaires de la wilaya, les associations civiles et les organisations écologiques qui doivent veiller au contrôle régulier de l'environnement de l'élevage et à l'intervention opportune lorsque cela est nécessaire.

\section{Références bibliographiques}

Becker B., 1997. Sustainability assessment: a review of values, concepts, and methodological approaches. Issues in Agriculture 10 - Washington, DC, Consultative Group on International Agricultural Research/World Bank (10), http://documents. worldbank.org/curated/en/564151468739297507/ Sustainability.

Bockstaller C., Cariolle M., Galan M-B., Guichard L., Leclercq C., Morin A., Surleau-Chambenoit C., 2013. Evaluation agri-environnementale et choix 
des indicateurs : acquis, enjeux et pistes. Innovations Agronomiques, 31: 1-14.

Bonaudo T., Lossouarn J., 2010. Aviculture et territoire : conditions d'un mariage durable ? In: $10^{\text {éme }}$ Journée des Productions Porcines et Avicoles, 13 Octobre 2010, Agro Paris Tech. Gembloux, Belgique, 34 p., http://gembloux.ulg.ac.be/.

Chiffoleau Y., 2010. Formes et enjeux des circuits courts de commercialisation en agriculture. Montpellier SupAgro CEP Florac, 37 p., http://www1. montpellier.inra.fr/,

Coudurier B., Blesbois E., 2013. Filières avicoles. In: Coudurier B., Georget M., Guyomard H., Huyghe C., Peyraud J.L., Vers des agricultures à hautes performances. Volume 4, Analyse des voies de progrès en agriculture conventionnelle par orientation productive. Ed. INRA, 278-293.

Coudurier B., Blesbois E., 2014. Filière volailles de chair. Analyse des voies de progrès en agriculture conventionnelle. Ed., INRA, France, 16 p.

Fortun-Lamothe L., 2011. Choix des indicateurs. Avantages et limites de la transformation en scores. Choix de la note finale de durabilité, Séminaire AVIBIO-9 décembre 2011-Angers.

Fortun-Lamothe L., 2012. L'évaluation de la durabilité des systèmes de production avicoles et cunicoles : principes, démarche, résultats et enseignements, 8 pages, http://www.facw.be/.

Fortun-Lamothe L., Litt J., Coutelet G., 2011. Evaluation de la durabilité des ateliers d'élevage DIAMOND (IDEA). Séminaire AVIBIO - 9 décembre 2011 - Angers, $47 \mathrm{p}$.

Gallot S., 2011. Différentes approches de l'évaluation du temps de travail sur les exploitations d'élevage appliquées à l'aviculture, "9 9 ème Journées de la Recherche Avicole, Tours, 29 et 30 mars $2011 », 5$ p.

Ghozlane F., Yakhlef H., Allane M., Bouzida S., 2006. Evaluation de la durabilité des exploitations bovines laitières de la Wilaya de Tizi-Ouzou (Algérie). New Medit, 4: 48-52.
Guillaumin A., Dockes A.C., Palazon R., 2009. La contribution des exploitations d'élevage au développement durable : état des lieux des méthodes d'évaluation et résultats. Renc. Rech. Ruminants, 16: 85-92.

Guyomard H., Huyghe C., Peyraud J.L., Boiffin J., Coudurier B,. Jeuland F., Urruty N., 2013. Vers des agricultures à hautes performances. Conception et évaluation de systèmes innovants en agriculture conventionnelle, Volume 2, Ed. INRA, $234 \mathrm{p}$.

Jez C., 2009. La filière avicole française à l'Horizon 2020 : Eléments de réflexion prospective. INRA, Unité prospective. Huitièmes Journées de la Recherche Avicole, St Malo, 25 et 26 mars 2009, 11-16.

Marta-Costa A.A., 2010. Sustainability study for the rearing of bovine livestock in mountainous areas. New Medit, 9(1): 4-12.

Richaud I., Rivard H,. Véron O., Blanchard E.B., 2015. Elevage et climat. Comprendre le problème, évaluer les solutions, Rapport scientifique de l'AVF, 32 p., http://www.defi-veggie.fr/.

Vilain L., Boisset K., Girardin P., Guillaumin A., Mouchet C., Viaux P., Zahm F., 2008. La méthode IDEA: Indicateurs de Durabilité des Exploitations Agricoles - Guide d'utilisation. Dijon : Ed. Educagri, $3^{\text {ème }}$ édition, $184 \mathrm{p}$.

Yakhelf H., Far Z., Ghozlane F., Madani T., Marie M., 2008. Evaluation de la durabilité des systèmes agropastoraux bovins dans le contexte de la zone semi-aride de Sétif (Algérie). New Medit, 7(4): 3639.

Zahm F., Girardin P., Mouchet M., Viaux P., Vilain L., 2005. De l'évaluation de la durabilité des exploitations agricoles à partir de la méthode IDEA à la caractérisation de la durabilité de la "ferme européenne " à partir d'IDERICA, Colloque International Organisé par l'Université Paul Cézanne (Aix-Marseille III, France), Indicateurs Territoriaux du Développement Durable, 1 et 2 décembre 2005, www.hitpges.com. 
